PAPER

\title{
Investigation of gain optimization technique in Doppler ultrasound system
}

\author{
Tatsuro Baba* \\ Toshiba Medical Systems Corporation \\ (Received 14 February 2008, Accepted for publication 13 May 2008)
}

\begin{abstract}
In this paper the author describes a high sensitivity Doppler ultrasound system based on the principle of ensemble mean processing utilizing automatic gain optimization through system parameters. This technique allows a reduction of the physical size of the hardware and simplifies gain adjustment by reducing the gain range substantially. Such a system is not subject to Doppler ultrasound system artifacts such as the mirror effect and quantization.
\end{abstract}

Keywords: Doppler ultrasound system, Dynamic range, Gain compensation, Mirror effect, Quantization, Ensemble mean process

PACS number: 43.35.Yb [doi:10.1250/ast.30.67]

\section{INTRODUCTION}

The dynamic range of conventional Doppler ultrasound diagnostic systems is insufficient for some applications. The blood flow signal is not sufficiently sensitive, and artifacts caused by signal clutter degrade the Doppler image quality [1,2]. In recent years, diagnostic ultrasound equipment performance has improved through the use of high-frequency electronics and integrated circuits. New diagnostic techniques are used because of the higher dynamic range available in both $\mathrm{B}$-mode imaging and blood flow analysis [3-7]. However, a higher dynamic range also requires more complicated gain control. It is possible to automatically optimize gain through the use of ultrasound system parameters. This technique reduces the size of the hardware and reduces the gain control range substantially.

\section{DOPPLER SIGNAL PROCESSING AND ITS DYNAMIC RANGE}

\subsection{Signal Processing}

Conventional Doppler ultrasound signal processing is shown in Fig. 1. The transceiver processor (Tx/Rx Proc.) receives signals from the probe elements and the received signals are amplified by the preamplifier. Gain compensation is applied to the signal to correct it for range-distance attenuation (STC: sensitivity time control), and an analog gain correction of the probe characteristics (frequency, sensitivity, etc.) is applied. The signal is then sent to an analog/digital converter (ADC). After AD conversion a

\footnotetext{
*e-mail: baba@us.nasu.toshiba.co.jp
}

digital beam former (DBF) applies a delay pattern to the data to focus it and produce beam data. This data is processed by tomogram processing (B-Mode Image Proc.) and Doppler signal processing (Doppler Image Proc.), then is displayed as a tomogram image and/or a spectrum Doppler image in the display processor (Display). The signal-to-noise ratio ( $\mathrm{S} / \mathrm{N}$ ratio) is increased in the $\mathrm{DBF}$ as the number of channels (corresponding to transducer elements) to which delay calculations are applied increases. In Doppler signal processing, quadrature detection (Mixer) is applied to the DBF output, and a band-pass filter (BPF) provides band limitation and rejects clutter. The result is a base-band Doppler signal. At this time, the $\mathrm{S} / \mathrm{N}$ ratio is sharply increased because of the band limitation of the BPF. In pulse-wave Doppler signal processing, we apply range-gate $(R G)$ integration across the range direction of the region of interest (ROI). This also increases the $\mathrm{S} / \mathrm{N}$ ratio.

In the case of a continuous-wave Doppler signal processing, the dynamic range is even larger and the high-pass filter (HPF) applied to this data must be more sophisticated. The dynamic range of the signal leaving the HPF is also much larger than that in the case of pulse-wave Doppler, on the order of $100 \mathrm{~dB}$ [8]. After fast Fourier transform (FFT) the $\mathrm{S} / \mathrm{N}$ ratio is greatly increased because of the butterfly integration. The dynamic range of the signal is now very large, and considerable gain adjustment and display compression must be performed in order to display the data.

\subsection{Dynamic Range and $\mathrm{S} / \mathrm{N}$ Ratio}

It is also necessary to take into consideration the fact 


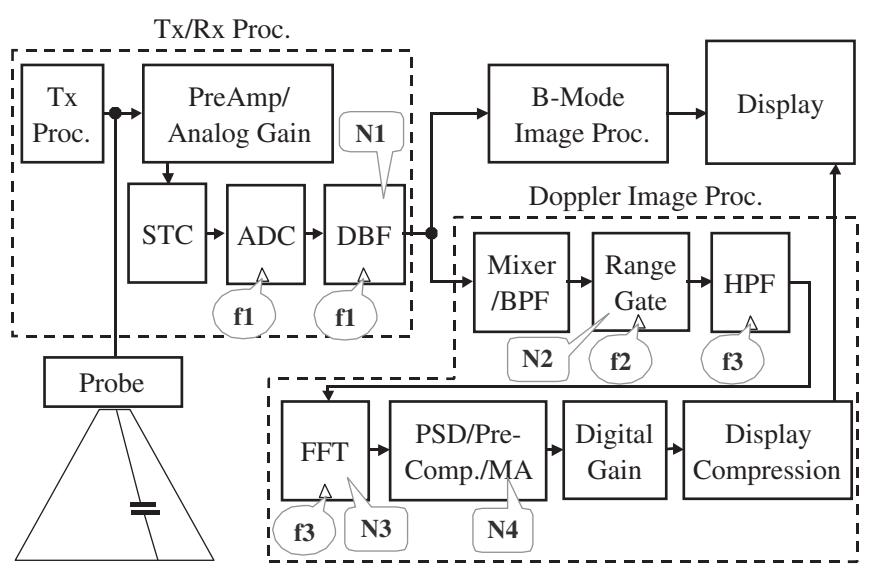

Fig. 1 Doppler ultrasound system.

that the dynamic range is increased in Doppler signal processing. In addition, a mirror effect and/or quantization artifacts are introduced when performing automatic gain compensation. Although the beam data leaving the DBF has a frequency of about $10-100 \mathrm{MHz}$, it is resampled at about $1-100 \mathrm{kHz}$. Thus, the input dynamic range of the FFT is increased by the band limitation effect. Moreover the $\mathrm{S} / \mathrm{N}$ ratio of the FFT output is increased in a similar manner to that resulting from ensemble mean processing $[9,10]$. The standard deviation of the noise increases inversely with the square root of the sample size $N$ according to

$$
\sigma_{z}^{2}(t) \cong \frac{1}{N} \sigma_{x}^{2}(t)
$$

$\sigma_{z}:$ standard deviation of output noise

$\sigma_{x}$ : standard deviation of input noise

Figure 2 shows a model of the $\mathrm{S} / \mathrm{N}$ ratio increase induced by ensemble mean processing. The integration of multiple channels ( $N$ channels) by the DBF and the band limitation by Mixer/BPF of Fig. 1 behave in a similar manner and can be treated similarly using Eq. (1). If the

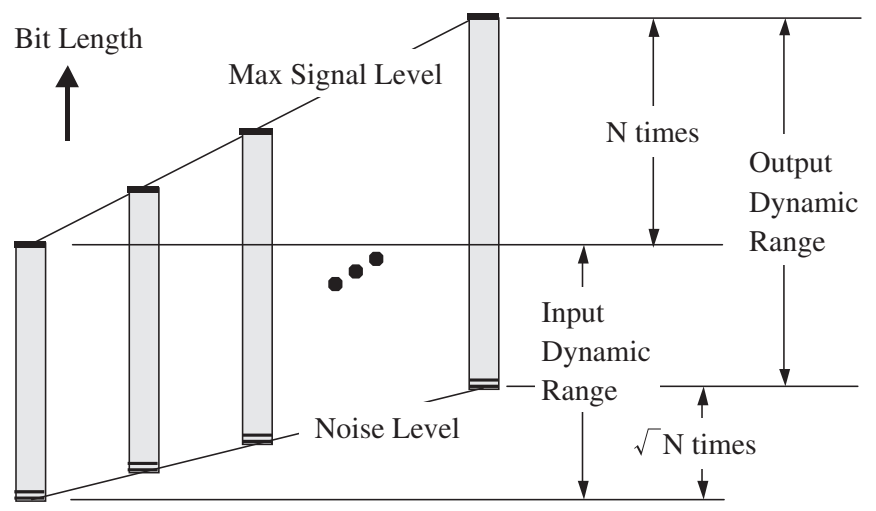

Fig. 2 Effect of the ensemble mean process. ensemble mean is set to $N$ (or the band limitation ratio is $1 / N)$, the maximum signal level will increase in proportion to $N$, but the noise level increases in proportion to $\sqrt{N}$. Thus, the $\mathrm{S} / \mathrm{N}$ ratio is increased a factor of $\sqrt{N}$.

\section{THE PURPOSE OF GAIN ADJUSTMENT}

Gain adjustment corrects the diagnostic target and Doppler sensitivity, and the effects resulting from differences in users' skills. In addition, it compensates for variations of other equipment parameters, such as the number of summing channels in the DBF, the apodization function, the bandwidth of the BPF, the integration length of RG, the FFT number, the window function, and the number of shift additions of the power spectrum depending on sweep speed [11-13]. The maximum signal level and the noise level vary with changes in these equipment parameters. In order to realize highly sensitive Doppler blood-flow diagnosis without saturation, a system with a high dynamic range must perform gain compensation taking all these parameters into account. Table 1 shows a rough estimation of the dynamic range and $\mathrm{S} / \mathrm{N}$ ratio based on the virtual system.

A model of the signal processing method accompanied by an increase in the $\mathrm{S} / \mathrm{N}$ ratio is shown in Fig. 3(a). The noise level and maximum signal level of the incoming signal are increased by signal processing. However, the increase in the noise level differs from that in the maximum signal level, and the overall $\mathrm{S} / \mathrm{N}$ ratio is increased. Under optimal gain adjustment (the range shown in light gray in Fig. 3(a)), there is no saturation of the maximum signal level, and the quantizing noise and the signal are not mixed in the output. When gain adjustment is unsuitable (the range shown in dark gray in Fig. 3(a)), a mirror effect or quantization artifacts occur in the spectrum image due to saturation or omission.

For signal amplitude, when the quantization accuracy is inadequate, quantizing noise is mixed with the signal [14-16]. A method of gain adjustment that takes the margin into consideration when detecting the weak Doppler signal around system noise and has sufficient quantizing margin is required. The effect of quantization is shown in Fig. 3(b), which shows the spectrum obtained when inputting a sinusoidal signal $\left(0.02 f_{\mathrm{s}}\right)$ including white noise. The horizontal axis is time and the vertical axis is frequency normalized by the sampling frequency $f_{\mathrm{s}}$. The quantizing level of the input range was changed every $2 \mathrm{~s}$ and was assigned values of 3, 5, 9 and 17. It turns out that the harmonic components $(-20$ to $-30 \mathrm{~dB})$ were produced by quantization with frequencies near $-0.3 f_{\mathrm{s}},-0.2 f_{\mathrm{s}}$, and $+0.25 f_{\mathrm{s}}$.

The mirror effect is an imaginal image symmetrically generated with a real image on both sides of a baseline [17]. In an analog system, it is mainly caused by the phase 
Table 1 Estimation of the increases in $\mathrm{S} / \mathrm{N}$ ratio and digital gain control range.

\begin{tabular}{|c|c|c|c|c|}
\hline Module & Cause & $\begin{array}{l}\text { Effect of D.R. } \\
\text { increment }^{* 1}\end{array}$ & $\begin{array}{l}\text { Conventional system } \\
\text { Fig. 4(a) (dB) }\end{array}$ & $\begin{array}{l}\text { New system } \\
\text { Fig. } 4(b)(d B)\end{array}$ \\
\hline ADC output & (Analog gain) & $50 \mathrm{~dB}$ & DR1@ @ $f_{1}$ & $\mathrm{DR} 1 @ f_{1}$ \\
\hline DBF & $\begin{array}{l}\text { Beam sum effect } \\
\left(N_{1} \text { channel }\right)\end{array}$ & $+50 \mathrm{~dB}$ & $\begin{array}{c}\mathrm{DR} 2 \\
=\mathrm{DR} 1+20 \log \left(N_{1}\right)\end{array}$ & $\begin{array}{c}\text { DR2_opt } \\
=\mathrm{DR} 1+20 \log \left(\sqrt{N_{1}}\right)\end{array}$ \\
\hline Mixer/BPF & $\begin{array}{l}\text { Band limitation effect } \\
\qquad\left(f_{1} / f_{2}\right)\end{array}$ & $+30 \mathrm{~dB}$ & $\begin{array}{c}\mathrm{DR} 3 \\
=\mathrm{DR} 1+\mathrm{DR} 2+20 \log \left(f_{1} / f_{2}\right)\end{array}$ & $\begin{array}{c}\text { DR3_opt } \\
=\mathrm{DR} 1+\text { DR2_opt }+20 \log \left(\sqrt{\left(f_{1} / f_{2}\right)}\right)\end{array}$ \\
\hline RG & $\begin{array}{l}\text { RG integration effect } \\
\qquad\left(N_{2} \text { tap }\right)\end{array}$ & $+40 \mathrm{~dB}$ & $=\begin{array}{c}\mathrm{DR} 4 \\
=\mathrm{DR} 1+\mathrm{DR} 2+\mathrm{DR} 3+20 \log \left(N_{2}\right)\end{array}$ & $\begin{array}{c}\text { DR4_opt } \\
=\text { DR1 + DR2_opt + DR3_opt + } \\
20 \log \left(\sqrt{N_{2}}\right)\end{array}$ \\
\hline FFT & $\begin{array}{c}\text { FFT processing effect } \\
\text { [FFT number and window] } \\
\quad\left(N_{3} \text { sampling }\right)\end{array}$ & $+50 \mathrm{~dB}$ & $=\frac{\mathrm{DR} 5}{\mathrm{DR} 1+\mathrm{DR} 2+\mathrm{DR} 3+\mathrm{DR} 4+20 \log \left(N_{3}\right)}$ & $\begin{array}{c}\text { DR5_opt } \\
=\mathrm{DR} 1+\text { DR2_opt }+ \text { DR3_opt }+ \text { DR4_opt } \\
+20 \log \left(\sqrt{N_{3}}\right)\end{array}$ \\
\hline PSD/Pre-Compres. & $\begin{array}{l}\text { Power dimension to } \\
\text { amplitude dimension }\end{array}$ & - & DR5 & DR5_opt \\
\hline MA & $\begin{array}{l}\text { Moving average effect } \\
\qquad\left(N_{4} \text { average }\right)\end{array}$ & $+10 \mathrm{~dB}$ & $=\begin{array}{c}\mathrm{DR} 6 \\
20 \log \left(N_{4}\right)\end{array}$ & $\begin{array}{c}\text { DR6_opt } \\
=\mathrm{DR} 1+\text { DR2_opt }+ \text { DR3_opt }+ \text { DR4_opt } \\
+ \text { DR5_opt }+20 \log \left(\sqrt{N_{4}}\right)\end{array}$ \\
\hline \multirow{3}{*}{ Digital gain } & Digital gain input & $\begin{array}{l}\text { D.R. before } \\
\text { digital gain input }\end{array}$ & $\begin{array}{r}230 \mathrm{~dB} \\
(\mathrm{DR} 6)\end{array}$ & $\begin{array}{l}140 \mathrm{~dB} \\
\left(\mathrm{DR} 6 \_\mathrm{opt}\right)\end{array}$ \\
\hline & Digital gain output & $\begin{array}{c}\text { D.R. after } \\
\text { digital gain output }\end{array}$ & $\begin{array}{l}\mathbf{7 0 ~ d B} \\
(\mathrm{DR} 7)\end{array}$ & $\begin{array}{l}70 \mathbf{d B} \\
(\mathrm{DR} 7)\end{array}$ \\
\hline & Gain control range & - & $\begin{array}{c}160 \mathbf{d B} \\
(\mathrm{DR} 6-\mathrm{DR} 7)\end{array}$ & $\begin{array}{c}\mathbf{7 0} \mathbf{d B} \\
\text { (DR6_opt-DR7) }\end{array}$ \\
\hline
\end{tabular}

${ }^{* 1}$ This estimation is based on virtual model of the Doppler ultrasound system.

error of quadrature detection or by small gain differences between IQ signals. In a digital system, although these effects do not occur, they are generated by a mirror effect owing to saturation. As shown in Fig. 3(c), a symmetrical mirror effect occurs on both sides of $0 \mathrm{~Hz}$ in the spectrum image. Figure 3(c) shows the image of spectrum in which the gain is raised $6 \mathrm{~dB}$ every $2 \mathrm{~s}$ up to the sinusoidal input including white noise. In this figure the FFT input dynamic range is 16 bit. The horizontal axis is time and the vertical axis is the frequency normalized by the sampling frequency $f_{\mathrm{s}}$. The mirror-effect component $\left(-0.2 f_{\mathrm{s}}\right)$ of the original signal component $\left(+0.2 f_{\mathrm{s}}\right)$ occurs as a result of saturation.

Thus, in conventional ultrasound design, both the mirror and quantization artifacts are caused by insufficient dynamic range of the system during Doppler signal processing.

\section{DESIGN OF SIGNAL PROCESSING SYSTEM}

Table 1 depicts the increases in the dynamic range and gain-control range of the conventional system and the new system based on the signal processing block diagram shown in Fig. 1. The DBF sums the beams of the $N_{1}$ channels. The Mixer/BPF limits the band-width into $f_{2} / f_{1}$. The RG integrates $N_{2}$ taps. The FFT integrates $N_{3}$ taps weighted by the window and operator. The PSD/PreComp. simply transfers the dimension (from amplitude to power) by applying the square root. The MA calculates the moving average of $N_{4}$ columns according to the sweep speed of the spectrum display.

Figure 4 shows the gain charts of the conventional system and new system based on Table 1 . The gain chart of the conventional system, which does not take the realization scale of the hardware into consideration, is shown in Fig. 4(a). In this study, we developed a system that can reduce the gain-control range as well as the internal dynamic range. Automatic gain compensation according to changes in the ranges of system parameters is realized for every subblock of the Doppler signal processing and is accompanied by an improved $\mathrm{S} / \mathrm{N}$ ratio. Since the ranges of the system parameters are known, the improvement of the $\mathrm{S} / \mathrm{N}$ ratio, the maximum signal level, and the noise level are calculable. The internal dynamic range and the gain adjustment range can be optimally designed for every subblock. By connecting partially optimized subblocks in series and uniting them, the internal dynamic range of the system can be reduced, thus, the system size and the total gain control range can both be sharply reduced. As shown in the example in Fig. 2, the internal $\mathrm{S} / \mathrm{N}$ ratio is increased by a factor of $\sqrt{N}$. Then, supposing the input signal 

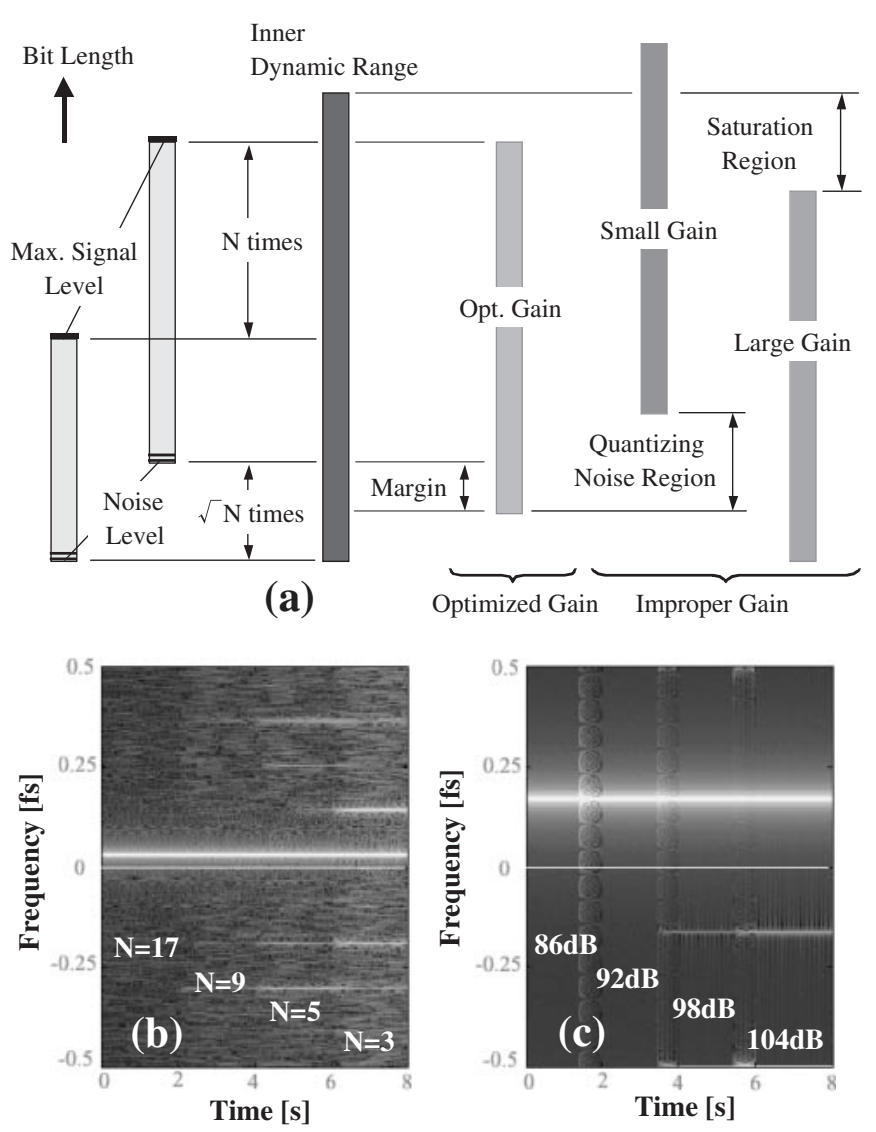

Fig. 3 Artifacts caused by inadequate gain control. (a) Problems of inadequate gain control. (b) Artifacts caused by quantization. (c) Artifacts caused by mirror effect.

dynamic range is DRin $[\mathrm{dB}]$, a range increase equivalent to $20 \log (\sqrt{N})$ [dB] will occur. Moreover, the internal dynamic range DRproc $[\mathrm{dB}]$, adds a margin of more than $(20 \log (\sqrt{12}))$ to the quantizing noise, can be roughly calculated using

$$
\text { DRproc } \geq \text { DRin }+20 \log (\sqrt{N})+20 \log (\sqrt{12}) .
$$

When digitizing, the least significant bit (LSB) must be rounded up or down not truncated, otherwise an error of $\mathrm{LSB} / 2$ will occur. The RMS value of the quantizing noise is equivalent to $\mathrm{LSB} / \sqrt{12}$. Thus, an additional margin of $\sqrt{12}$ for the dynamic range must be maintained so that rounding can be performed accurately [18]. Although the internal dynamic range DRproc is only sufficient for automatic gain compensation using the system parameters, it is necessary to consider a margin for the original gain adjustment that compensates for the diagnostic target and Doppler sensitivity variations in an internal dynamic range. The gain chart of the subblock signal processing accompanied by range expansion is shown in Fig. 4(b). The gaincontrol range of the conventional system (Fig. 4(a)) is DR6-DR7, and the gain-control range of the new system (Fig. 4(b)) is DR6_opt-DR7. In general Doppler signal

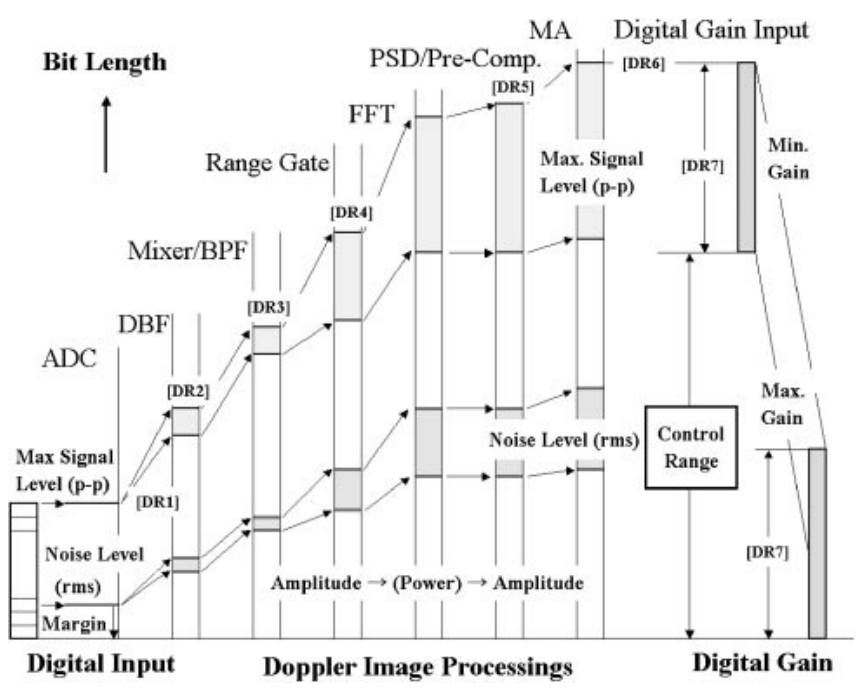

(a)

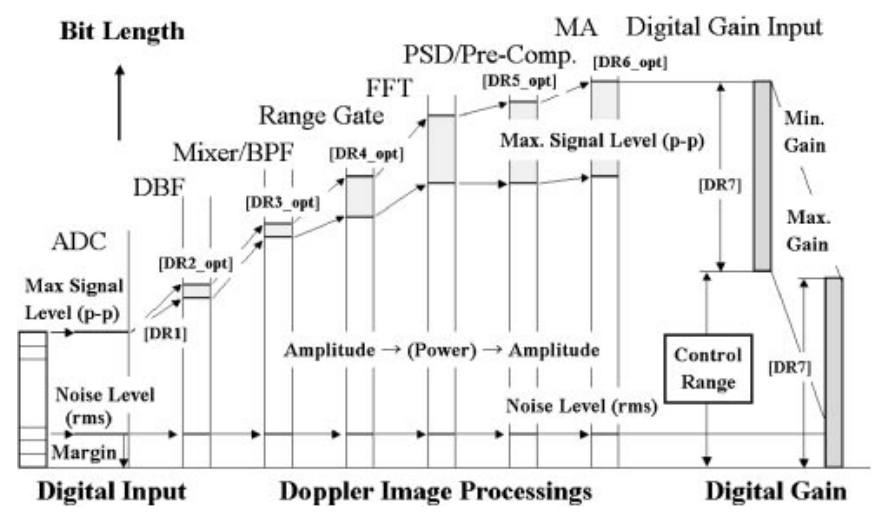

(b)

Fig. 4 Comparison of gain control systems. (a) Gain chart of the conventional system. (b) Gain chart of the new system.

processing, DR6 is above $200 \mathrm{~dB}$ and DR7 (the digital gain output) is the display luminance range (about $70 \mathrm{~dB}$ ). Thus, the gain-control range of the conventional system should be more than $130 \mathrm{~dB}$, which is very large. On the other hand, DR6_opt for new system is smaller than DR6, about $100 \mathrm{~dB}$. We can reduce not only the gain-control range but also the inner dynamic ranges of the submodules at the same time.

\section{RESULT}

The effect of automatic gain optimization using the new system was verified in a simulation of RG integral processing. Spectra of the conventional system and the new system when changing the RG width are shown in Figs. 5(a) and 5(b), respectively. The horizontal axis is time and the vertical axis is the frequency normalized by sampling frequency $f_{\mathrm{s}}$. The RG was adjusted from $1 \mathrm{~mm}$ to $4 \mathrm{~mm}$ then to $16 \mathrm{~mm}$ in $1.8 \mathrm{~s}$ intervals. A sinusoidal signal including white noise was used as the input. In the 

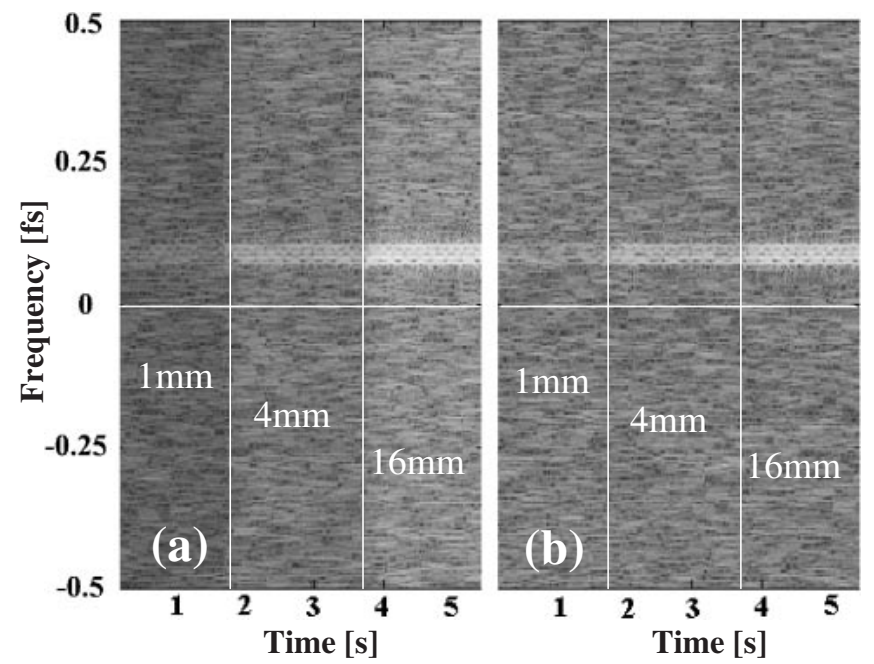

Fig. 5 Effect of automatic gain compensation: example of RG integration process. (a) Conventional system. (b) New system.

conventional system, the signal level and noise level increase with increasing RG width. For this reason, the user should reduce gain manually when the RG width is increased. In the new system, although the signal level rises if the RG width is increases, it turns out that the noise level does not change. As mentioned above, by Doppler automatic gain compensation, the input bit length of each signal processing block can be made smaller, and the gain adjustment range can also be decreased as necessary.

\section{CONCLUSION}

Through the use of ensemble mean processing it is possible to reduce the overall dynamic range of an ultrasound signal while maintaining signal quality. As a result, the bit depth required to represent the signal can be reduced, resulting in smaller and more cost-effective system hardware. Furthermore, gain adjustments of the operator and the final system are simplified meaning that less operator adjustment is required. The system does not generate the mirror and quantization artifacts that occur in conventional ultrasound system designs.

\section{REFERENCES}

[1] T. Baba, "Investigation of wall filter in Doppler ultrasound system,” Soc. Signal Process. Appl. Technol. Jpn., 9(2), pp. 1419 (2006) (in Japanese).

[2] T. Baba, "Evaluation of post wall filter for Doppler ultrasound system," in Acoustical Imaging, Vol. 29, I. Akiyama, Ed. (Springer Science + Business Media, Berlin, 2008), pp. 133138.
[3] J. Lisauskas, J. Singh, M. Courtois and S. J. Kovacs, "The relation of the peak Doppler E-wave to peak mitral annulus velocity ratio to diastolic function," Ultrasound Med. Biol., 27, 499-507 (2001).

[4] C. Bruch, R. Gradaus, S. Gunia, G. Breithardt and T. Wichter, "Doppler tissue analysis of mitral annular velocities," J. Am. Soc. Echocardiography, 16, 1031-1036 (2003).

[5] C. W. Guron, M. Hartford, A. Persson, J. Herlitz, D. Thelle and K. Caidahl, "Timing of regional left ventricular lengthening by pulsed tissue Doppler," J. Am. Soc. Echocardiography, 17, 307-312 (2004).

[6] H. Kanai and Y. Koiwa, "Myocardial rapid velocity distribution," Ultrasound Med. Biol., 27, 481-498 (2001).

[7] T. Oki, Y. Oishi and T. Ishimoto, "Tissue Doppler imaging," J. Med. Ultrason., 30, 639-650 (2003) (in Japanese).

[8] J. A. Jensen, Estimation of Blood Velocities Using Ultrasound: A Signal Processing Approach (Cambridge University Press, London, 1996), pp. 146-153.

[9] The Physical Society of Japan, Experimental Data Processing Using Computer (Saiensu-sha Co., Ltd., Tokyo, 1987), pp. 8892 (in Japanese).

[10] G. Date, A. V. Oppenheim and R. W. Schafer, Digital Signal Processing (Corona Publishing Co. Ltd., Tokyo, 1981), pp. 45-48 (in Japanese).

[11] K. Ranganathan and W. F. Walker, "A novel beamformer design method for medical ultrasound. Part I: Theory," IEEE Trans. Ultrason. Ferroelectr. Freq. Control, UFFC-50, 15-24 (2003).

[12] M. Nagano and T. Nakata, "Frequency sweep spectrum analyzer," Soc. Signal Process. Appl. Technol. Jpn., 3(4), pp. 17-24 (2000) (in Japanese).

[13] K. Kubo, "Method for estimating instantaneous frequency and parameters of sine-wave by differential domain sampling," Trans. Soc. Instrum. Control Eng., 42, 845-853 (2006) (in Japanese).

[14] K. Miyawaki, Noise Analysis (Asakura Publishing Co. Ltd., Osaka, 1978), pp. 14-23 (in Japanese).

[15] Y. Kasahara, Information Theory and Communication Method (Kyoritsushuppan Co., Ltd., Tokyo), 1976, pp. 204-211 (in Japanese).

[16] S. Minami and S. Kawada, The Introduction of Data Processing for Science Measurement (CQ Pub. Co., Ltd., Tokyo, 2002), pp. 88-92 (in Japanese).

[17] H. Susaki, " $I / Q$ sampling method as pre-processing: Frequency analysis," Soc. Signal Process. Appl. Technol. Jpn., 5(3), pp. 2-7 (2002) (in Japanese).

[18] V. Cappellini, A. G. Constantinides and P. Emiliani, Digital Filters and Their Applications (Academic Press, London, 1978), pp. 166-184.

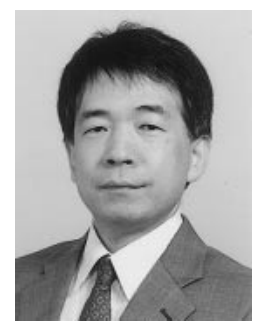

Tatsuro Baba received the B.S., M. Sc. in precision engineering from Osaka University, and Ph.D. degree in Mechanical engineering from Kobe University in 1981, 1983 and 2006, respectively. From 1983 has been employed at Toshiba Medical Systems Corporation, Tochigi. His research interests include Doppler ultrasound system and digital signal processing. 